\title{
Correction to: The Examined Life is Wise Living: The Relationship Between Mindfulness, Wisdom, and the Moral Foundations
}

\section{Paul Verhaeghen $^{1}$ (D)}

Published online: 21 January 2021

๑) Springer Science+Business Media, LLC, part of Springer Nature 2021

\section{Correction to: Journal of Adult Development (2020) 27:305-322 https://doi.org/10.1007/s10804-019-09343-y}

The original version of the article has contained some errors in text and tables.

The errors are given below and the original article has been updated.

Participants: Reliability estimates: For Sample A: median $=0.75$; for Sample B: median $=0.85$, ranging from .57 to .93$)$

Measures and Procedure-Wisdom Scales: Reliability of reflective subscale of $3 \mathrm{D}-\mathrm{WS}=0.80$ and 0.85 ; of affective subscale $=0.75$ and 0.83 .

Results-Factor Analysis of the Wisdom Scales: Intercorrelations between the two factors $=0.47$ and 0.14 .
Discussion-The Structure of Wisdom: Next to last sentence ("The correlation between ... of 0.11 ") to be deleted.

Discussion-Predicting Wisdom About the (Social) World: Two-sentence section ("There is a hint ... reached significance") to be deleted.

Discussion-Predicting Wisdom About the (Social) World: Percentage in variance of wisdom explained about the (social) world $=5-29 \%$.

Discussion-Wisdom and the Moral Foundations: Beta values for the final step in explaining the binding foundation from social conservatism $=0.52$ and 0.75 ; from wisdom about the self $=0.25$ and 0.14 ; and from wisdom about the (social) world -0.07 and -0.01 .

The correct Tables 1, 2, 3, and 4 are given below.

The original article can be found online at https://doi.org/10.1007/ s10804-019-09343-y.

Paul Verhaeghen

verhaeghen@gatech.edu

1 School of Psychology, Georgia Institute of Technology, 654

Cherry Street NW, Atlanta, GA 30320, USA 
Table 1 Factor analysis of the nine wisdom scales in both samples; principal axis analysis with oblimin rotation

\begin{tabular}{|c|c|c|c|c|}
\hline & \multicolumn{2}{|l|}{ Sample A } & \multicolumn{2}{|l|}{ Sample B } \\
\hline & $\begin{array}{l}\text { Factor } 1 \\
\text { Wisdom about } \\
\text { the self }\end{array}$ & $\begin{array}{l}\text { Factor } 2 \\
\text { Wisdom about the } \\
\text { social world }\end{array}$ & $\begin{array}{l}\text { Factor } 1 \\
\text { Wisdom about } \\
\text { the self }\end{array}$ & $\begin{array}{l}\text { Factor } 2 \\
\text { Wisdom about } \\
\text { the social world }\end{array}$ \\
\hline ASTI (total) & .55 & & .76 & \\
\hline SAWS-emotion regulation & .62 & & .72 & \\
\hline SAWS-experience & .75 & & .69 & \\
\hline SAWS-humor & .56 & & .73 & \\
\hline SAWS-openness & .44 & & .68 & \\
\hline SAWS-reminisce-reflect & .77 & & .65 & \\
\hline 3D-WS-affective & & .71 & & .77 \\
\hline 3D-WS-cognitive & & .65 & & .59 \\
\hline 3D-WS-reflective & & .64 & & .91 \\
\hline
\end{tabular}

$N=260$ for Sample A and 173 for Sample B. For legibility reasons, factor loadings below .30 are not represented 


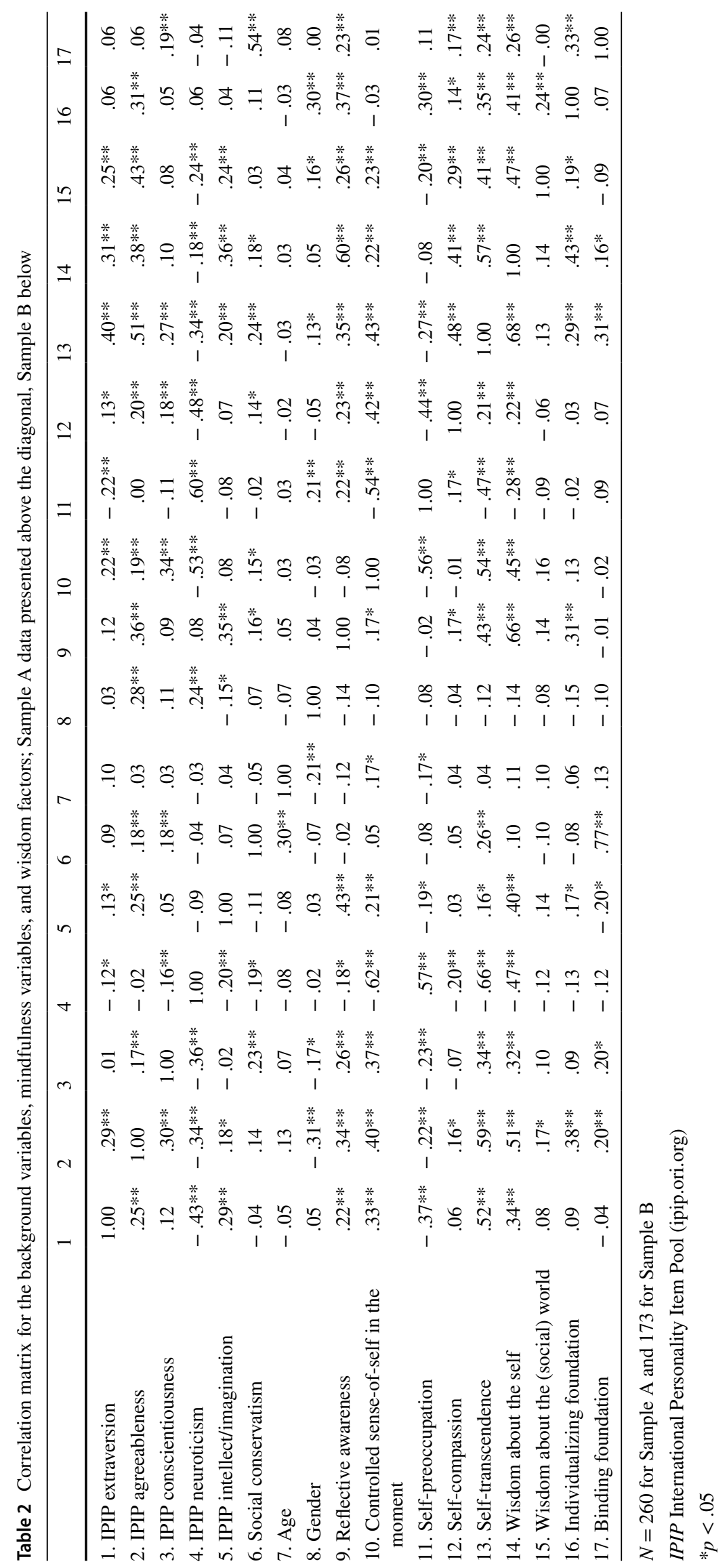


Table 3 Results from hierarchical regression analyses to predict the wisdom factors

\begin{tabular}{|c|c|c|c|c|c|c|c|c|}
\hline & \multicolumn{2}{|l|}{ Step 1} & \multicolumn{2}{|l|}{ Step 2} & \multicolumn{2}{|l|}{ Step 3} & \multicolumn{2}{|l|}{ Step 4} \\
\hline & Sample A & Sample B & Sample A & Sample B & Sample A & Sample B & Sample A & Sample B \\
\hline \multicolumn{9}{|l|}{ Wisdom about the self } \\
\hline IPIP extraversion & $0.19 * *$ & 0.08 & $0.16^{* *}$ & 0.02 & $0.17 * *$ & 0.03 & $0.11^{*}$ & -0.06 \\
\hline IPIP agreeableness & $0.24 * *$ & $0.26 * *$ & 0.08 & $0.17 * *$ & 0.06 & $0.17 * *$ & -0.01 & 0.05 \\
\hline IPIP conscientiousness & 0.01 & $0.07 *$ & -0.06 & 0.01 & -0.06 & 0.03 & -0.08 & 0.02 \\
\hline IPIP neuroticism & $-0.16^{* *}$ & $-0.21 * *$ & $-0.15^{* *}$ & $-0.19^{* *}$ & -0.10 & $-0.17 *$ & -0.06 & -0.05 \\
\hline IPIP intellect/imagination & $0.28 * *$ & $0.31 * *$ & $0.13 * *$ & 0.11 & $0.16^{* *}$ & 0.11 & $0.14 *$ & $0.18 * *$ \\
\hline Age & -0.01 & 0.08 & -0.02 & $0.13 *$ & -0.01 & $0.12 *$ & 0.01 & $0.13^{*}$ \\
\hline Gender & 0.07 & -0.06 & 0.08 & 0.01 & 0.07 & 0.02 & 0.05 & 0.02 \\
\hline Reflective awareness & & & $0.52 * *$ & $0.50 * *$ & $0.46 * *$ & $0.49 * *$ & $0.40 * *$ & $0.38 * *$ \\
\hline Controlled sense-of-self in the moment & & & $0.15 *$ & 0.12 & 0.12 & 0.13 & 0.07 & 0.09 \\
\hline Self-preoccupation & & & & & 0.04 & -0.01 & 0.05 & 0.05 \\
\hline Self-compassion & & & & & $0.19 * *$ & 0.06 & $0.14 *$ & 0.03 \\
\hline Self-transcendence & & & & & & & $0.28 * *$ & $0.41 * *$ \\
\hline$R^{2}$ & .296 & .455 & .506 & .622 & .526 & .625 & .561 & .673 \\
\hline$R^{2}$ change & $.296^{* *}$ & $.455^{* *}$ & $.210 * *$ & $.167 * *$ & $.020 * *$ & .003 & $.035 * *$ & $.048 * *$ \\
\hline \multicolumn{9}{|l|}{ Wisdom about the (social) world } \\
\hline IPIP extraversion & 0.10 & 0.05 & 0.09 & 0.04 & 0.08 & 0.04 & 0.06 & 0.04 \\
\hline IPIP agreeableness & $0.33 * *$ & 0.08 & $0.29 * *$ & 0.07 & $0.28 * *$ & 0.08 & $0.26 * *$ & 0.08 \\
\hline IPIP conscientiousness & -0.04 & 0.01 & -0.05 & -0.01 & -0.05 & -0.04 & -0.06 & -0.04 \\
\hline IPIP neuroticism & $-0.25^{* *}$ & -0.03 & $-0.25 * *$ & -0.01 & $-0.19 *$ & -0.06 & $-0.18 *$ & -0.07 \\
\hline IPIP intellect/imagination & 0.15 & 0.12 & 0.11 & 0.10 & 0.11 & 0.10 & 0.10 & 0.10 \\
\hline Age & 0.02 & 0.09 & 0.01 & 0.09 & 0.03 & 0.10 & 0.03 & 0.10 \\
\hline Gender & $0.14^{*}$ & -0.04 & $0.14 *$ & -0.03 & $0.15^{*}$ & -0.03 & $0.14 *$ & -0.03 \\
\hline Reflective awareness & & & $0.11 *$ & 0.06 & $0.16^{*}$ & 0.07 & 0.14 & 0.08 \\
\hline Controlled sense-of-self in the moment & & & 0.12 & 0.05 & -0.02 & 0.05 & -0.04 & 0.06 \\
\hline Self-preoccupation & & & & & -0.12 & 0.07 & -0.12 & 0.07 \\
\hline Self-compassion & & & & & 0.05 & -0.10 & 0.03 & -0.10 \\
\hline Self-transcendence & & & & & & & 0.10 & -0.01 \\
\hline$R^{2}$ & .286 & .053 & .302 & .056 & .312 & .064 & .316 & .064 \\
\hline$R^{2}$ change & $.286^{*}$ & .053 & .016 & .003 & .010 & .008 & .004 & .000 \\
\hline
\end{tabular}

$N=260$ for Sample A and 173 for Sample B

IPIP International Personality Item Pool (ipip.ori.org)

${ }^{*} p<.05 ; * * p .01$ 


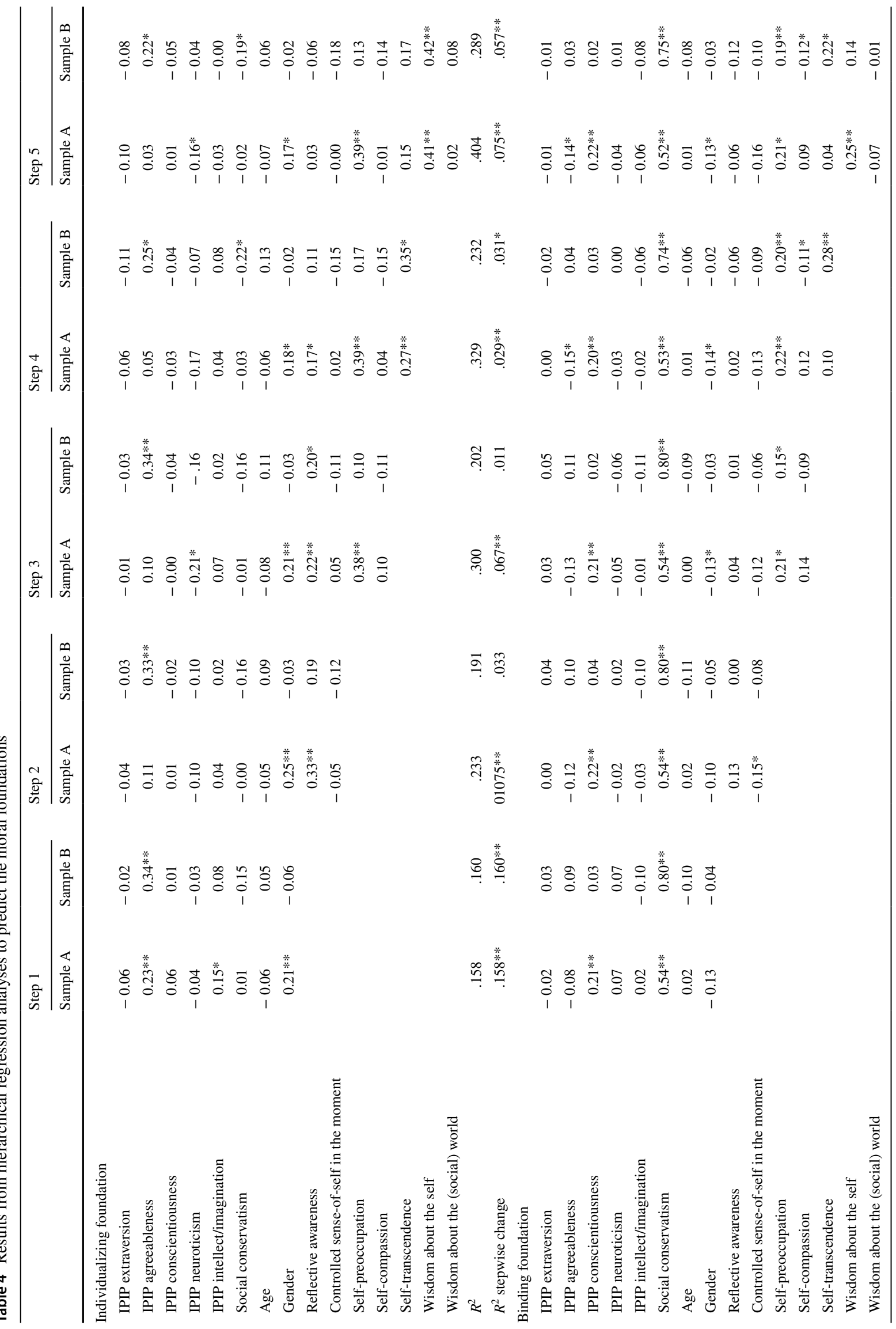




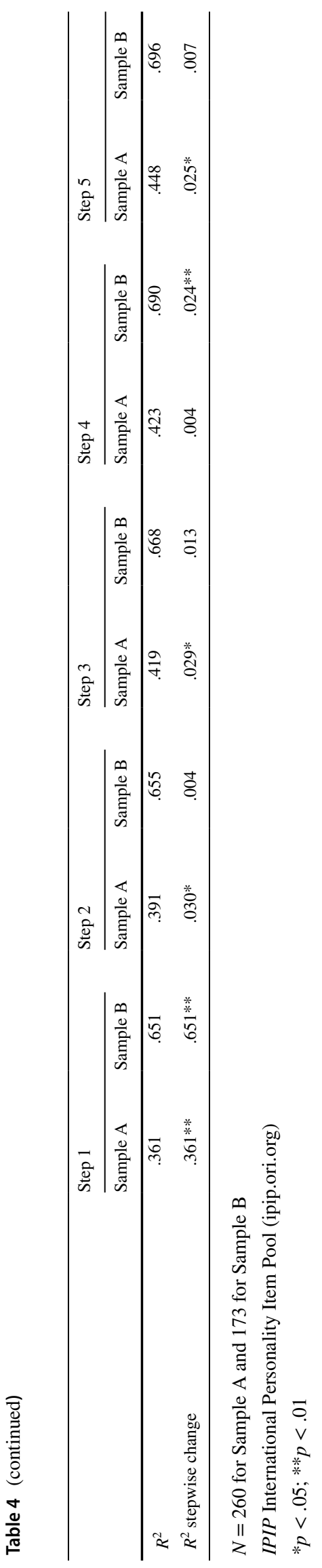

Publisher's Note Springer Nature remains neutral with regard to jurisdictional claims in published maps and institutional affiliations. 